\title{
Motivational component of educational activity in the context of a new educational network paradigm
}

\author{
Elena Rudenko ${ }^{1}$, Elizaveta Sagajdachnaya ${ }^{2 *}$, and Karina Shamraeva ${ }^{2}$ \\ ${ }^{1}$ Don State Technical University, 344003, Rostov-on-Don, Russia \\ ${ }^{2}$ Rostov State Economic Universiry, 344007, Rostov-on-Don, Russia
}

\begin{abstract}
The creation of a digital environment is currently a priority in the state policy concerning the educational sphere. This is due to the digitalization of social life in all its manifestations. Today, researchers from various scientific fields are searching for ways to create an adequate model of digital learning that can form the conditions for successful educational activities. This article examines the role of the motivational component in the learning process in the digital educational environment. The change in the educational paradigm, which has arisen as a result of the influence of digitalization of human activity, determines the dominant characteristics of the student's personality, leading to success in the implementation of educational and work activities. It is established that there is a need to study not only the means and material base of digital learning, but also its essential foundations, including the role of competencies and personal qualities of students. The article describes the competencies of the student that are important for effective learning activities. The role of the teacher in the development of motivation for learning is considered. The directions of pedagogical activity that are significant for the formation of motivation for learning are determined. The analysis of the conditions of digitalization of education has shown that the successful formation of the motivational component of training is possible with a combination of traditional and innovative pedagogical methods.
\end{abstract}

\footnotetext{
* Corresponding author: sag-natali@yandex.ru
} 


\section{Introduction}

The global scientific community is showing an increased interest in such a problem as the digitalization of education. Researchers study the impact of the digital environment in this area in a comprehensive way: from the fundamental principles of digital learning to the set of tools and platforms through which this learning is implemented in practice [1]. Scientists seek to trace the history of the development of e-learning [digital learning] [2] and to systematize the changes in education associated with digitalization [3], to identify the features of the manifestation of the qualities of a representative of the "Net Generation" in learning [4], to identify the problems and advantages of online learning [online learning] [5], to present a critical analysis of the process of digitalization in education $[6,7]$.

The problem of digitalization of education is devoted to dissertation research, scientific articles covering various areas of secondary and higher education. Russian authors consider the main directions, problems and prospects of developing a digital educational environment $[8,9]$, a competence-based approach to digital education [10], technological means for the successful implementation of digital learning [11], the economic aspect of digitalization of education $[12,13]$, etc. In these studies, the main focus is on the study of tools that can be applied in practice for the implementation of a digital education project. The essence of digitalization as a component of the educational process, its place in the system of educational values, and the interaction of the digital educational environment with other components of the structure of educational activity are not considered in so much detail in Russian science. We can name the works of scientists who study the impact of digitalization on the development of mental processes of students' personality $[14,15,16]$, issues of competence formation in training in the digital environment [17], etc.

Of course, the study of the tools of digital learning at this stage of the introduction of digitalization in education comes to the fore, because in the context of the rapidly increasing need for the use of high technologies in the transfer of knowledge, it is necessary to identify the most effective ways of learning. But the definition of strategies for the further development of the digital educational environment, its impact on students, on the formation of their knowledge system, on the development of mental processes, etc. They are no less necessary, since this will allow us to design the most effective system of digital education, which could be integrated into schools, secondary vocational and higher educational institutions. This is possible only in the process of a deep and detailed study of the essential basis of the phenomenon of digitalization of education.

The most relevant issues, in our opinion, are the interaction of the principles of digital education with the principles of traditional education, the possibility of including the components of the emerging educational paradigm in the existing paradigm. Such issues include the role of a teacher in the learning process, the development of a student's personality, the formation of his / her competencies, etc. The need for such research is due to the functioning of continuity in education. This article contributes to the study of the features of actualization of changes in the motivational component of learning in the context of a 
change in the educational paradigm. Digitalization of the learning process involves strengthening the independent work of students with educational material and a certain decrease in the share of teacher participation in this process, while increasing the role of a teacher in the control of assimilation knowledge and creating a motivational basis for learning activities. It is assumed that the human factor, i.e. the psychological aspect of the educational process, remains the dominant position in achieving the learning result, only the learning tools and the set of key competencies change. In particular, the article presents a study of the specifics of the motivational component in the new conditions of education. This problem is one of the most acute among teachers who implement digital learning in their practice.

Digitalization of education is considered in various scientific fields (philosophy, economics, cultural studies, etc.), but the study of the problem of the introduction of digital technologies in educational activities from the point of view of pedagogy is able to determine how digitalization will affect the development of a harmonious personality of a student, whether it will be possible and in what conditions.

\section{Materials and Methods}

The purpose of this study is to consider possible changes that occur in the process of implementing a new educational paradigm: strategies for the development of a new educational environment, teacher-student interactions, and most importantly, changes in the motivational component of learning.

The research is based on the theoretical issues of Russian and foreign scientists in the field of philosophy and methodology of education. The study of the problem of digitalization of education in the conditions of modern society was carried out within the framework of systematic and dialectical approaches, which are determined by its purpose and objectives. The interdisciplinary approach is justified by the study of the digital educational environment from the point of view of philosophy, sociology and pedagogy.

In the course of the work, the method of system analysis was applied, based on the study of the educational process as a system of interacting components. The method of ascent from the abstract to the concrete is used in the study of the influence of the principles of the formation of a digital educational model on the mechanisms of its individual components. In addition, when studying the problem of motivation to learn, the analysis of psychological and pedagogical literature related to this problem was used. When identifying possible prospects for digitalization of learning, the method of modeling the socio-cultural process was used.

Thus, the methods of generalization, system analysis, synthesis and comparison are used in the work.

The empirical objects of research are groups of sources: legislative initiatives and federal projects that effect the main trends in the development of digitalization of modern schools; theoretical and practical works of domestic and foreign researchers (over the past 20 years) devoted to the peculiarities of the introduction of digitalization in the educational process, personal changes of the object and subject of education in the digital environment. 


\section{Results}

The study of the specifics of the digital environment in Russian education is based on the existence of the Federal projects "Modern School", "Digital Educational Environment", "Digital School", which set out a program for creating a digital educational environment, expected to be completed in 2024. Digitalization should cover all stages of learning: from the acquisition of primary knowledge by students to the control of their assimilation. The report "Problems and Prospects of digital transformation of education in Russia and China" substantiates several tasks that must be achieved for the implementation of these projects. The primary task is the development of material infrastructure (the creation of data centers, new communication channels, providing students with devices necessary for the use of digital educational materials). The material resources also include electronic programs used in the learning process. Material resources should be applied during the gradual development of online learning. This means that online learning is not introduced with the simultaneous abandonment of the traditional form: these forms coexist, and training with paper sources decreases over time. Online learning is built in a learning management system that is designed with the goals and objectives of digital education in mind. This system includes programs for the administration and control of training courses.

Creating an effective online learning system should include a number of tools for creating a safe environment, so it is planned to develop a system of universal student identification. In addition, for the effectiveness of online learning, it is necessary to ensure the availability of formed ICT competencies. The implementation of these tasks is designed to construct models of an educational institution, which should take into account the interaction of all components of digital learning.

In addition to these tasks, the process of forming an ideal model of the digital educational environment should include the issue of forming the personality of the student and his / her competencies in the conditions of online learning. In this case, the process of adaptation to learning in the digital environment, which has a special socio-pedagogical significance, is also important. The development of the methodological basis for the integration of the subject of education not only in the new learning process, but also in the new, "digital" society should be carried out in the aspect of a systematic approach, which takes into account not only the material conditions and means of digital education, but, first of all, the personal qualities of a student, personal values and beliefs that serve to create a system of ideas about society. Of course, the formation of the student's personality is influenced both by the digital environment in which he receives education and also by various factors of social life, for example, globalization processes. In addition, mass media, Internet communications, social networks, etc. have an impact [18].

As a result, there is a need to develop a methodological framework that would ensure the development of a harmonious personality of the student, who has formed critical thinking, creative potential, and high social activity. The creation of an integral system of forms of digitalization of the educational process means the orderly integration of its forms in the 
subsystems of education at all levels and stages into a holistic methodological system with clearly defined characteristics, logical structure and the process of its modeling in the system of continuing education.

It is necessary to take into account that the implementation of the program for the digitalization of education takes place at the time of a change in the educational paradigm, which is due to "socio-cultural transformations caused by the post-industrial, information culture, which is full of sources of information and requires students to be able and willing to extract it, rather than receive it ready-made, the ability to use it creatively" [19].

If the paradigm is understood as a generalizing model of methodological, theoretical and applied activities of the scientific community, then at the moment in the educational sphere there is a development and expansion of a personality-oriented paradigm aimed at solving creative tasks, activating the independent activity of students, using the principles and means of problem-based learning.

Researchers define the new educational paradigm as a "network educational paradigm" [20], in the center of which is a student - a representative of the "network generation". This educational paradigm can also be defined as a "digital educational paradigm", if we proceed from the designation of the means of learning and the way of thinking of a representative of the new digital society. The transition from one paradigm to another in any field, including education, is associated with numerous difficulties that have only just begun to be understood by scientists. There is still a long process of forming a paradigm, during which a new education system will be formed with a full methodological justification of all the components of this system. Modern education is witnessing the birth of a completely new educational paradigm, in which the student is endowed with hitherto unknown opportunities to independently determine and build their own educational trajectory [19].

The education system in the conditions of the new network (digital) educational paradigm belongs to the anthropic-socio-cultural systems [21], i.e., systems in which the substrate is heterogeneous, combining the characteristics and properties of nature, society and human activity. The complexity of the new educational system is to build a methodology based on the synthesis of real, objective reality and virtual reality, rational consciousness and intuitive principle. This factor determines the construction of training as the creation of new knowledge on the basis of existing ones with the involvement of all these aspects of the training system. The attention of the participants of the educational process should be directed, first of all, to the development of abilities and motivation to generate their own ideas. At the heart of the new educational paradigm, the emergence of which is due to digitalization, the student is considered from the standpoint of the development of his her key competencies, so the main task of education is to develop the ability to think outside the box, find new solutions to the task, creatively process the available information, i.e. from the relay of knowledge, the learning process moves to the generation of this knowledge.

In this case, the development of independence in learning, the ability to self-organize comes to the fore. However, the development of independence in the digital educational environment is called into question, which is due to the properties of a digital system that can 
act independently of the user, assumes the presence of analytical and predictive functions, where the user is necessary for setting the problem, and its solution may remain outside of his competence. As a result, there is a contradiction: whether digitalization is a condition for developing the student's independence or counteracts this process.

The role of the learner in the aspect of developing independence radically changes its character: now he needs to take on certain tasks, the implementation of which will allow not only to manage his own educational activities, but also to be responsible for its results, i.e. part of the activities to control the formation of competencies is assigned to the learner him herself. The role of a teacher is also changing. It is assumed that teaching activities are divided into two areas: subject-based learning activities and accompanying activities. If the first direction, in general, is traditional - it is integrated into the digital environment - then the second direction for the support of trainees is relatively new. The activity of a teachermentor consists in helping students to work in new educational conditions: in organizing activities, in achieving above-subject results, in developing the necessary personal qualities, in monitoring the assimilation of knowledge, that is, in improving the quality of education. The teacher-mentor helps to create a stable motivation for students to conduct activities. In addition, the teacher-mentor coordinates joint work with parents and other "significant adults". The motivational aspect of learning in a digital environment is evaluated by a number of authors as an advantage of a new form of education: it is noted that digitalization contributes to the education of students 'independence, since" without excessive tutelage of teachers, the student will achieve higher results" and will have an understanding that it is necessary to act independently in a digital environment [8]. However, this thesis is quite controversial. The fact is that a person immersed in the digital environment is exposed to negative processes. So, according to the statement of E. Fromm, the technological improvement of society, of individual spheres of human activity, has a destructive effect on the person living within this society: a person becomes passive, does not feel his her own identity, as a result of which helshe loses integrity [22]. In addition, due to the loss of the significance of cultural meanings, a person does not get used to a new picture of the world, does not make it part of the life experience, but focuses on the information interpreted by others. The result is a loss of self-identity. As a result, the primary task is to form a new identity to replace the lost one. If we talk in this aspect in relation to the trainees as representatives of the network generation, then in this case it is not necessary to restore cultural meanings, since the self-identity of children is not yet formed, and teachers need to work with the values of digital culture. As a result of this approach, it will be possible to overcome the trend of negative impact of digitalization on students.

Motivation to learn can be defined as a natural reaction to learning opportunities, which increases in the following cases: 1) students' self-developed assessment of the educational material; 2) independent determination of the significance of a particular learning opportunity; 3) awareness of their own freedom in the implementation of learning; 4) understanding of their own ability to learn [23].

Factors such as challenge, curiosity, engagement, and control contribute to increased 
motivation $[24,25]$. Based on this statement, the researchers found that increasing motivation is influenced by the level of complexity of the task performed: the more complex the task, the more interesting it is for the student, since in this case, such motivational bases as challenge and curiosity are activated [26]. Of course, the complexity of the task should correspond to the potential capabilities of the student-attempts to solve a deliberately impossible task will lead to a decrease in interest and motivation. It is necessary to involve tasks that require a heuristic algorithm for their implementation, implying the presence of several solutions, and including the possibility of creative search.

J. M. Keller's model of increasing motivation in e-learning (ARCS model) includes several components: attention, relevance, confidence, satisfaction [27]. Attention involves the involvement of students using multimedia material that contains interesting graphics, animation, or any other means that can arouse interest. Students' attention can also be attracted through material that offers a problem with a non-standard solution and variability. The variability of the task is necessary, because the student adapts and loses interest in the task over time if the predictability of the storyline is planned [28].

Relevance as a component of motivation involves providing students with consistent goals based on their past experience and skills expected to be obtained, as well as compatible with their individual learning styles. This factor is related to Goal Theory, which determines that setting goals motivates behavior. These can be learning or achievement goals, and they can be proximal (achieved in a reasonably short time) or distal (achieved in the distant future). In addition to setting clear goals, students should be kept informed of their progress towards achieving these goals [29].

Confidence as a component of motivation involves helping students from the teacher to establish expectations of success. This factor is related to the theory of self-efficacy (selfefficacy theory], i.e. a student must believe that helshe is able to act in a certain way to achieve the educational goal. In addition, the confidence factor is related to the Attribution Theory, i.e., how the student explains their successes and failures. The student can attribute the success or failure of the task to him herself or to external causes. According to the ARCS Model, a teacher should make an effort to help students relate their learning outcomes to what they can control themselves.

Satisfaction involves designing learning so that learners get a positive experience from their learning experience. This factor may be related to the Expected Value Theory, which states that students expect certain results from behavior. The more the student values the results, the more likely it is that their motivation will increase. This component includes the following motivational strategies:

- verbal reinforcement, reward, personal attention;

- feedback and conscious avoidance of negative influences [29].

This idea is framed in Figure 1 [30]. 


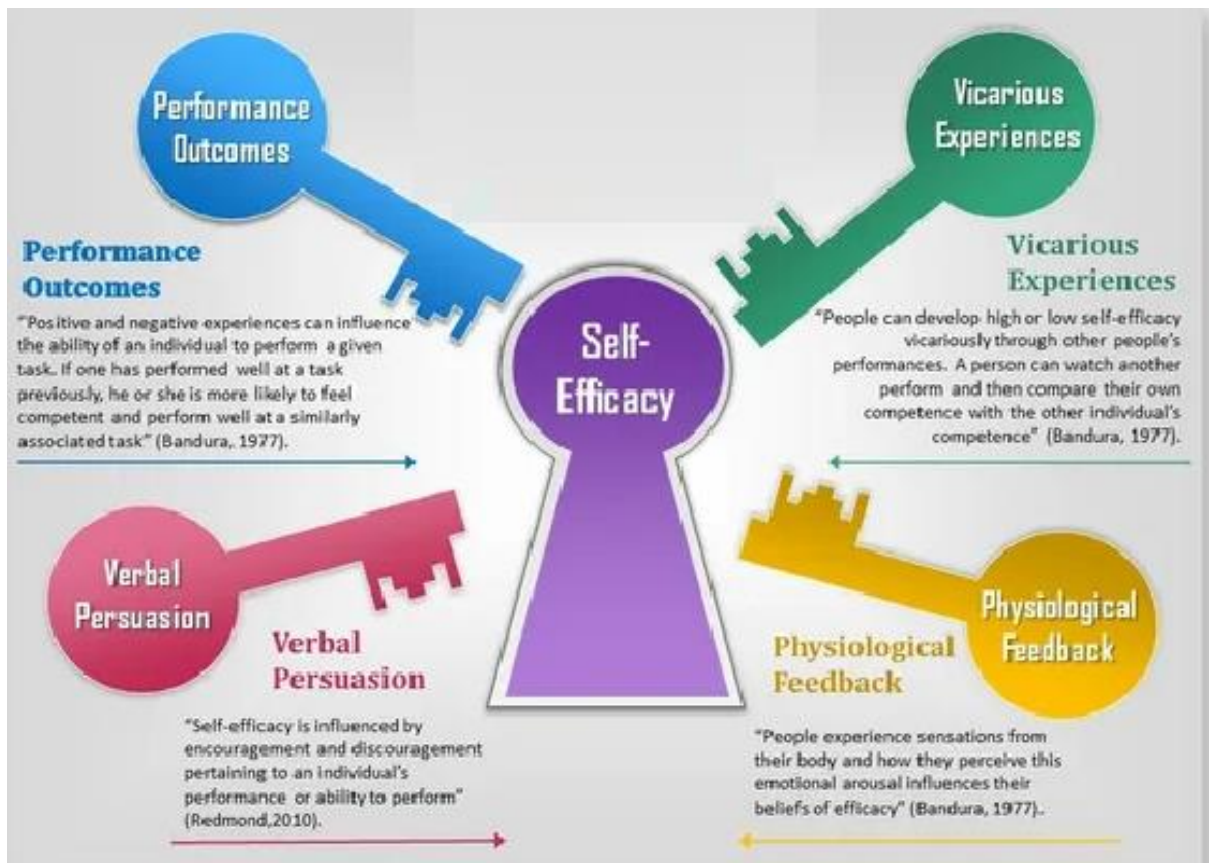

Fig. 1. Four factors of determining efficacy judgments.

The digital environment is new to the perception of students, so it is difficult for selfdevelopment, high activity and the development of independence. E-learning leads to positive results - a high level of academic performance and the development of thinking, if it contains a high level of activity of students and their involvement in the learning process anytime, anywhere. It is noted that students tend to be less involved in the distance learning environment.

E-learning is more effective than traditional e-learning, as the degree of interaction between students and teachers decreases [31]. Distance hinders communication, which forces students to participate continuously and effectively in online learning, i.e. distance is a factor that reduces motivation to learn. In this regard, it is necessary to develop tools that allow the teacher to interact with students to the extent that the situation requires.

Student engagement in online learning is defined as the level of effort that students put into interacting with learning resources over a certain period of time, resulting in experience and improved academic performance [31]. When students are engaged in their learning, they can improve their academic achievements and then apply the knowledge they have gained in real life. Student engagement is also an indicator of the quality of education and a fundamental condition for success in academic and future professional activities. Many of the tools used by teachers to increase motivation to learn in a digital environment remain 
unchanged and depend on the age of the students. Thus, in primary and secondary school age, game technologies significantly increase the motivation for the perception of a particular educational information, but they acquire a special form - electronic (this can be materials prepared by the teacher himself, or selected by him with the help of Internet resources). Students of middle and high school age, as well as university students, perceive the Internet primarily not as an educational platform, but as a socio-cultural phenomenon that allows them to conduct a variety of activities (cultural, social, entertainment, etc.), which significantly increases interest in any information provided by various websites, including educational ones. In addition, high school students and university students have a sufficiently developed critical and logical thinking, which allows them to use the acquired knowledge for their reproduction and generation of new ones, creating their own projects. To implement their ideas, trainees strive to find as much additional information as possible, which develops the skill of searching for it and embedding it in the existing picture of the world. Consequently, the activities carried out in this way affect the formation of a thinking, creative personality capable of carrying out independent educational and work activities.

Considering the concept of motivation, scientists argue that students of all ages are able to self-motivate and manage their own learning if they work with educational tasks that they perceive as interesting, personally significant or relevant [23].

As a rule, this means that the activities carried out are associated with explicit or hidden personal goals. The need to increase motivation to learn appears in cases where: 1) the learning task is not of interest to students, does not correspond to their personal goals and attitudes; 2) students do not have the ability to control the process of learning; 3) students believe that they lack the knowledge or skills to solve a particular task; 4) students lack adequate external support and resources, including the help of a teacher, respect and encouragement from a significant adult.

If the task is perceived by a student as uninteresting, boring, tedious or meaningless, then the help of a teacher is required, who is able to change the student's attitude to the task or transform the task so that it seems attractive to solve.

So, if a student is interested in performing learning activities, the motivation to learn is stimulated naturally, since learning tasks are perceived as personally significant.

If a student's interest is absent, then the motivation to learn must be stimulated from the outside in order to overcome the lack of internal motivation caused by the perception of the learning task as having no personal meaning. The above can be applied both to the traditional form of learning and to the digital form.

There are also specific motivational characteristics that are unique to online learning. In this case, we are talking not only about special methods of presenting the material, but also about the specifics of the technical nature. Therefore, digital learning should take into account the fact that part of the time and effort the student will spend on learning the interface, so it should be made as simple as possible. The fact that the technical side of the question is directly related not only to the successful assimilation of the material, but also to the motivation for learning in general does not require proof. 
In this case, the role of the teacher is great, whose task is not only to build an effective motivational system that would allow students to successfully integrate into the digital environment, but, in addition to knowledge, skills and abilities, the formation of which takes place directly in the learning process using computer tools, to transfer to students a system of values and certain experience, which repeatedly increases the responsibility of the teachers and imposes new additional requirements on them. The role of a teacher of the traditional educational system shows its inefficiency, the change of the role of the passive performer to the active participant and a doer of the process meets a certain amount of resistance from teachers who are not ready, not willing to open up to new trends and developments [32].

Thus, digital culture cannot be a component that completely replaces the educational culture, but should become only a part of it, complementing the existing educational sphere and contributing to the education of a harmonious personality of the student. In the new paradigm of education, digitalization is a component that directly affects the work of the educational system, the nature of the action of each component. Digitalization affects the features of interaction between the main participants of the educational process - the student and the teacher, whose positions should be reviewed in the light of changes in the educational paradigm. However, the fundamental nature of the relationship between the participants of the pedagogical process remains the same and is based on the psychological and pedagogical features of this process.

Thus, there is an interaction of four educational phases [33] that transform the modern educational space of the student: adopting, adapting, transforming, transferring.

Adopting refers to certain competencies that improve the skills of both an individual student and a group as a whole. Adapting is a kind of modification of the experience without changing the basic structure. Transforming is the restructuring of the experience gained. Transferring is understood as the creation of channels for the transfer of new professional skills, involving other members of the group/team in the adoption, adapting, transforming (see Fig. 2).

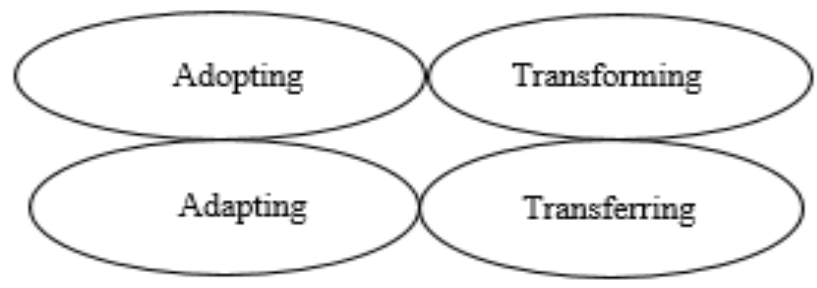

Fig. 2. Phases of Transformative Education.

Digitalization of education leads to the predominance of such competencies of the student as: 1) the ability to find information in the shortest possible time; 2) the ability to process this information, introduce it into the body of knowledge; 3) the ability to critically comprehend the knowledge gained; 4) the ability to independently conduct work; 5) the ability to effectively interact with other people. When learning in the conditions of digital education, 
technological literacy and logical thinking are formed, which are necessary for working with the means of electronic education. The full development of these competencies and mental processes requires a rethinking of the motivational basis of training, in the construction of which the leading role belongs to the teacher who plays the role of a mentor.

Digital culture, combining traditional educational culture, forms new socio-cultural meanings and values. In it, the development of virtual type of communication is found, where the information flow destroys the traditional forms of personal identification. This trend should be overcome in the process of developing a new educational model in the context of digitalization of education.

\section{Discussion}

So, the ways of developing the motivational component in learning in the context of the integration of education into the digital social environment require revision and modeling in accordance with the requirements of the new educational paradigm. It is known that in the learning process (regardless of whether traditional means and technologies are used or digital), the problem of forming motivation and increasing interest in the educational material is one of the most urgent.

Digitalization of learning, on the one hand, has an advantage in this regard, since the process of acquiring new knowledge in an attractive digital environment is perceived positively by students. On the other hand, the network environment familiar to students contains a lot of information that does not carry a learning component, and with insufficiently developed critical thinking can be an obstacle to the assimilation of knowledge. In this case, the role of a teacher is invaluable, who performs the task of developing students' competencies necessary for the correct perception of the digital environment and the effective use of digital learning tools.

Consequently, the role of a teacher does not change significantly - only the emphasis on the formation of critical thinking, independence in work, and high motivation to generate new knowledge on the basis of the received ones is shifted. In addition, in pedagogical activity, the direction of mentoring is highlighted, the task of which is to accompany a student in the organization of educational activities.

These factors and mechanisms of action of the educational system in the new digital environment should correlate with the ideas about other components of this system, which will allow us to form a model of digital learning that can be successful in the conditions of modern Russian education. Of course, additional problems in research on the formation of motivation to learn can only be identified in practice, which may require a reassessment of the point of view on this issue.

\section{Conclusion}

From the above, we can conclude that the changes that appear in the process of introducing 
a new educational paradigm are not always positive. Despite the failure and inefficiency of the former role of a teacher from the traditional educational system in the new digital paradigm, the urgent need for qualitative changes in this role, the basic principle of the relationship between the participants of the pedagogical process remains the same and is based on the psychological and pedagogical features of this process.

It should be noted that the abundance of changes in the process of implementation of a new education paradigm are not always positive. Never the less the digitalization of educational activities in the context of human activity in all spheres of social life is inevitable, as is the inclusion of a digital component in the education system. Therefore, it is necessary to search for ways to overcome the negative trends that arise in the process of becoming digitalization and a new educational paradigm and strengthening positive trends. It is also necessary to search for new solutions in the organization of educational activities, ways to increase the interest of students in educational activities. The primary task in this case is the development of critical thinking in students and the development of independent work skills. The shift in emphasis in the motivation to learn in the new digital environment requires a new teacher to have the skills and abilities of "contextual" education, the purpose of which is to create a holistic socio-practical activity of a developed person, individual.

\section{References}

1. W.G. Bowen, Higher Education in the Digital Age (Princeton University Press, NY, 2013)

2. D. Kergel, Digital Learning in Motion: From Book Culture to the Digital Age (Routledge, NY, 2020)

3. M. Thomas, Digital Education: Opportunities for Social Collaboration (Springer, Luxembourg, 2011)

4. D. Tapscott, Growing Up Digital: The Rise of the Net Generation (McGraw-Hill, Chicago, 1999)

5. M.E. Milacovich, J.-M. Wise, Digital Learning: The Challenges of Borderless Education (Edward Elgar Publishing Limited, Northampton, 2019)

6. L. Gourlay, Posthumanism and the Digital University: Texts, Bodies and Materialities (Bloomsbury Publishing, London, 2020).

7. N. Negroponte, Being digital (Hodder \& Stoughton, London, 1995)

8. I.M. Azhmukhamedov, Prospects and priorities of teacher education in the era of transformations, choices and challenges 2, 3-12 (2020)

9. A.A. Strokov, Digital culture and values of Russian education: abstract of the $\mathrm{PhD}$ thesis (Nizhny Novgorod Institute of Management - branch of RANEPA, Nizhny Novgorod, 2021) 
10. T.E. Pakhomova, Formation of ICT competence of students of pedagogical college taking into account interdisciplinary integration in the conditions of digitalization of education (Abstract of the $\mathrm{PhD}$ thesis, Chita, 2020)

11. V.A. Traynev, Electronic educational resources in the development of the information society (generalization and practice) (Dashkov \& Co, Moscow, 2015)

12. M.L. Berkovich, Development of the market of higher education services in the conditions of digitalization (on the materials of the Chelyabinsk region) (Abstract of the PhD thesis, Simferopol, 2020).

13. E.M. Dorofeev, Bulletin of LOIRO 3, 72-76 (2019)

14. A.M. Kondakov, A.A. Kostyleva, Bulletin of the Peoples' Friendship University of Russia. Series: Informatization of education 16(2), 207-218 (2019)

15. N.S. Kramarenko, Bulletin of the Moscow State Regional University 3 191-201 (2020)

16. M.S. Yanitsky, Professional education in Russia and abroad 2, 38-44 (2019)

17. N.I. Sorokina, R.I. Stepanov, E.Yu. Popova, Pedagogical education in Russia 6, 24-29 (2019)

18. A.K. Oreshkina, The world of science 6(5), 34-34 (2018)

19. V.A. Testov, Bulletin of the Nizhny Novgorod State University, NO Lobachevsky 4, 150-56 (2012)

20. G.A. Berulava, The European Proceedings of Social \& Behavioural Sciences 4, 8-23 (2010)

21. M.S. Kagan, Voprosy filosofii 12, 13-18 (1996)

22. E. Fromm, The Heart of Man (American Mental Health Foundation, NY, 2010)

23. B.L. McCombs, Aurora 8-13 (1996)

24. R. Martens, J. Gulikers, and T. Bastiaens, Journal of Computer Assisted Learning 20(5), 368-376. Wiley. Retrieved from https://www.learntechlib.org/p/98581/.

25. M.J. Rosenberg, E-Learning Strategies for Delivering Knowledge in the Digital Age (McGrawHill, Chicago, 2000)

26. R.H. Shroff et al., Communications of the Association for Information Systems 19(1) (19), 241-260 (2007)

27. J.M. Keller, Journal of Instructional Development 10(3), 2-10 (1987)

28. J.M. Keller, K. Suzuki, Journal of Educational Media 29(3), 229-239 (2004)

29. C.B. Hodges, Journal of Interactive Online Learning 2(3), 1-7 (2004)

30. G. Lopez-Garrido, Simply Psychology (2020) https://www.simplypsychology.org/selfefficacy.html.

31. J. Lee, H.D. Song, A.J. Hong, Sustainability 11(4), 985-996 (2019)

32. Arbona Xhemajli., Engineering and Education 4(1), 31-37 (2016) 
33. M. Klarin, International Journal of Cognitive Research in Science, Engineering and Education 4(1), 67-71 (2016) 